\title{
FACTORIZATION TECHNIQUES FOR THE NONLINEAR MODEL OF QUASI-STATIONARY PROCESSES IN CRYSTALLINE SEMICONDUCTORS
}

\author{
Beatriz Juarez-Campos, Elena I. Kaikina, Pavel I. NAumkin \\ AND HECTOR F. RUIZ-PAREDES
}

\begin{abstract}
We consider the question of global existence and asymptotics of small solutions of a certain pseudoparabolic equation in one dimension. This model is motivated by the wave equation for media with a strong spatial dispersion, which appear in the nonlinear theory of the quasi-stationary processes in the electric media. We develop the factorization technique to study the large time asymptotics of solutions.
\end{abstract}

Mathematics subject classification (2010): 35B40, 35Q60.

Keywords and phrases: Nonlinear pseudoparabolic equation, global in time existence, Asymptotics of solutions, Factorization Techniques.

\section{REFERENCES}

[1] L. Abdelouhab, Nonlocal dispersive equations in weighted Sobolev spaces. Differential Integral Equations 5, 2 (1992), 307-338.

[2] L. Abdelouhab, J. Bona, M. Felland And J. SAut, Nonlocal Models for Nonlinear Dispersive Waves, Phys. D, 40 (1989), 360-392.

[3] J. P. Albert, J. Bona And D. B. Henry, Sufficient Conditions for Stability of Solitary-Wave solutions of model Equation for Long-Waves, Phys. 240 (1987), 343, 366.

[4] A. B. Al'shin, M. O. Korpusov And A. G. Sveshnikov, Blow-up in nonlinear Sobolev type equations. De Gruyter Series in Nonlinear Analysis and Applications, 15. Walter de Gruyter \& Co., Berlin, 2011. xii+648 pp.

[5] T. B. BenJAmin, J. L. BonA, AND MAHONY, Model equations for long waves in nonlinear dispersive systems, Philos. Trans. Royal Soc. London, Ser. A, 272 (1972), pp. 47-78.

[6] M. V. Fedoryuk, Asymptotic Methods in Analysis, in: Analysis. I. Integral representations and asymptotic methods. Encyclopaedia of Mathematical Sciences, 13. Springer-Verlag, Berlin, 1989. vi+238 pp.

[7] N. HAYASHI, Global existence of small solutions to quadratic nonlinear Schrödinger equations, Commun. P.D.E., 18 (1993), pp. 1109-1124.

[8] N. HaYASHI AND P. I. NAUMKIn, The initial value problem for the cubic nonlinear Klein-Gordon equation, Zeitschrift fur Angewandte Mathematik und Physik, 59, 6 (2008), 1002-1028.

[9] N. HAYASHI AND P. NAUMKIN, Factorization technique for the fourth-order nonlinear Schrödinger equation. Z. Angew. Math. Phys. 66, 5 (2015), 2343-2377.

[10] N. HAYASHI AND P. NAUMKIN, On the inhomogeneous fourth-order nonlinear Schrödinger equation. J. Math. Phys. 56, 9 (2015), 093502, 25 pp.

[11] N. HAYASHI AND P. I. NAUMKIn, Factorization technique for the modified Korteweg-de Vries equation. SUT J. Math. 52, 1 (2016), 49-95.

[12] N. HaYashi AND T. OzaWA, Scattering theory in the weighted $L^{2}\left(R^{n}\right)$ spaces for some Schrödinger equations, Ann. I.H.P. (Phys. Théor.), 48 (1988), 17-37.

[13] B. JuareZ-Campos, E. Kaikina, AND H. F. Ruiz-Paredes, Nonlinear model of quasi-stationary process in crystalline semiconductor. Differ. Equ. Appl. 9, 1 (2017), 37-55. 
[14] M. O. Korpusov, AND E. V. YushKov, Local solvability and blow-up for Benjamin-Bona-MahonyBurgers, Rosenau-Burgers and Korteweg-de Vries-Benjamin-Bona-Mahony equations, Electron. J. Differential Equations 2014, No. 69, 16 pp.

[15] I. P. NAUMKIN, Sharp asymptotic behavior of solutions for cubic nonlinear Schrödinger equations with a potential, Journal of Mathematical Physics 57, 051501 (2016); http://doi.org/10.1063/1.4948743.

[16] I. P. NAUMKIN, Initial-boundary value problem for the one dimensional Thirring model, Journal of Differential equations, 261, 8 (2016), 4486-4523.

[17] I.P. NAUmKIN, Nonlinear Schrödinger equations with exceptional potentials. J. Differential Equations, 265, 9 (2018), 4575-4631.

[18] E. M. SteIn AND R. ShAKARChi, Functional analysis. Introduction to further topics in analysis. Princeton Lectures in Analysis, 4. Princeton University Press, Princeton, NJ, 2011. xviii+423 pp.

[19] A. G. Sveshnikov, A. B. AL'shin, M. O. Korpusov, And Yu. D. Pletner, Linear and nonlinear equations of Sobolev type, (FIZMATLIT, Moscow, 2007), pp. 734. 